\title{
Front Matter: Volume 8714
}

, "Front Matter: Volume 8714," Proc. SPIE 8714, Radar Sensor Technology XVII, 871401 (17 June 2013); doi: 10.1117/12.2029726

SPIE Event: SPIE Defense, Security, and Sensing, 2013, Baltimore, Maryland, SPIE. United States 


\title{
PROCEEDINGS OF SPIE
}

\section{Radar Sensor Technology XVII}

\author{
Kenneth I. Ranney \\ Armin Doerry \\ Editors
}

29 April-1 May 2013

Baltimore, Maryland, United States

Sponsored and Published by

SPIE 
The papers included in this volume were part of the technical conference cited on the cover and title page. Papers were selected and subject to review by the editors and conference program committee. Some conference presentations may not be available for publication. The papers published in these proceedings reflect the work and thoughts of the authors and are published herein as submitted. The publisher is not responsible for the validity of the information or for any outcomes resulting from reliance thereon.

Please use the following format to cite material from this book:

Author(s), "Title of Paper," in Radar Sensor Technology XVII, edited by Kenneth I. Ranney, Armin Doerry, Proceedings of SPIE Vol. 8714 (SPIE, Bellingham, WA, 2013) Article CID Number.

ISSN: 0277-786X

ISBN: 9780819495051

Published by

SPIE

P.O. Box 10, Bellingham, Washington 98227-0010 USA

Telephone +1 3606763290 (Pacific Time) · Fax +1 3606471445

SPIE.org

Copyright (C) 2013, Society of Photo-Optical Instrumentation Engineers.

Copying of material in this book for internal or personal use, or for the internal or personal use of specific clients, beyond the fair use provisions granted by the U.S. Copyright Law is authorized by SPIE subject to payment of copying fees. The Transactional Reporting Service base fee for this volume is $\$ 18.00$ per article (or portion thereof), which should be paid directly to the Copyright Clearance Center (CCC), 222 Rosewood Drive, Danvers, MA 01923. Payment may also be made electronically through CCC Online at copyright.com. Other copying for republication, resale, advertising or promotion, or any form of systematic or multiple reproduction of any material in this book is prohibited except with permission in writing from the publisher. The CCC fee code is $0277-786 \mathrm{X} / 13 / \$ 18.00$.

Printed in the United States of America.

Publication of record for individual papers is online in the SPIE Digital Library.

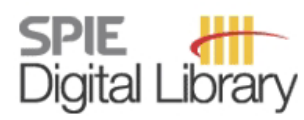

SPIEDigitalLibrary.org

Paper Numbering: Proceedings of SPIE follow an e-First publication model, with papers published first online and then in print and on CD-ROM. Papers are published as they are submitted and meet publication criteria. A unique, consistent, permanent citation identifier (CID) number is assigned to each article at the time of the first publication. Utilization of CIDs allows articles to be fully citable as soon as they are published online, and connects the same identifier to all online, print, and electronic versions of the publication. SPIE uses a six-digit CID article numbering system in which:

- The first four digits correspond to the SPIE volume number.

- The last two digits indicate publication order within the volume using a Base 36 numbering

system employing both numerals and letters. These two-number sets start with 00, 01, 02, 03, 04,

05, 06, 07, 08, 09, 0A, OB ... 0Z, followed by 10-1Z, 20-2Z, etc.

The CID Number appears on each page of the manuscript. The complete citation is used on the first page, and an abbreviated version on subsequent pages. Numbers in the index correspond to the last two digits of the six-digit CID Number. 


\section{Contents}

ix Conference Committee

\section{SESSION 1 SPECIAL SESSION: NOISE RADAR I}

871402 Human detection and ranging at long range and through light foliage using a W-band noise radar with an embedded tone [8714-1]

K. A. Gallagher, R. M. Narayanan, The Pennsylvania State Univ. (United States)

871403 FPGA implementation of a software-defined radar processor [8714-2]

H. Suarez, Y. R. Zhang, The Univ. of Oklahoma (United States)

871404 A compressive radar system with chaotic-based FM signals using the Bernoulli map [8714-3]

H. A. Ochoa, C. Teja Enugula, The Univ. of Texas at Tyler (United States)

\section{SESSION 2 SPECIAL SESSION: NOISE RADAR II}

871406 Demonstration of detection and ranging using solvable chaos [8714-5]

N. J. Corron, U.S. Army Aviation \& Missile Research, Development and Engineering Ctr.

(United States); M. T. Stahl, NASA Marshall Space Flight Ctr. (United States); J. N. Blakely,

U.S. Army Research, Development and Engineering Command (United States)

871407 Initial experimental results using the noise correlation radar [8714-6]

M. A. Govoni, U.S. Army Communications-Electronics Research, Development and

Engineering Ctr. (United States); J. Silvious, J. Clark, U.S. Army Research Lab. (United States);

C. Overman, Univ. of Florida (United States)

871408 Ranging and target detection performance through lossy media using an ultrawideband S-band through-wall sensing noise radar [8714-7]

S. Smith, R. M. Narayanan, The Pennsylvania State Univ. (United States)

871409 GNSS-based passive radar sensing using hybrid-aperture system [8714-8]

R. Silver, Y. R. Zhang, H. Suarez, Y. Pan, Y.-R. Huang, The Univ. of Oklahoma (United States)

\section{SESSION 3 APPLICATIONS: CONCEALED TARGETS I}

$8714 \mathrm{OB}$ Design of spectrally versatile forward-looking ground-penetrating radar for detection of concealed targets [8714-10]

B. R. Phelan, The Pennsylvania State Univ. (United States); M. A. Ressler, G. J. Mazzaro,

K. D. Sherbondy, U.S. Army Research Lab. (United States); R. M. Narayanan, The

Pennsylvania State Univ. (United States) 
8714 OC Development of a longer range stand off millimetre wave radar concealed threat detector [8714-11]

N. J. Bowring, M. J. Southgate, D. A. Andrews, N. D. Rezgui, S. W. Harmer, D. O'Reilly, Manchester Metropolitan Univ. (United Kingdom)

8714 OD Emulation of forward-looking radar technology for threat detection in rough terrain environments: a scattering and imaging study [8714-12]

D. Liao, T. Dogaru, U.S. Army Research Lab. (United States)

8714 OE Multitone harmonic radar [8714-13]

G. J. Mazzaro, A. F. Martone, U.S. Army Research Lab. (United States)

\section{SESSION 4 EMERGING TECHNOLOGIES}

8714 OG Radar tomography assisted three-dimensional localization via the noisy stepped frequency waveform [8714-67]

R. Vela, L. J. E. Bracken, Air Force Research Lab. (United States)

$8714 \mathrm{OH}$ Cognitive processing for nonlinear radar [8714-57]

A. Martone, K. Ranney, A. Hedden, G. Mazzaro, D. McNamara, U.S. Army Research Lab. (United States)

8714 ol Scattering and imaging of nonlinearly loaded antenna structures in half-space environments [8714-70]

D. Liao, U.S. Army Research Lab. (United States)

SESSION 5 APPLICATIONS: CONCEALED TARGETS II

8714 0J Effect of pulse fidelity on detection of landmines [8714-19]

H. Öztürk, H. Nazlı, TÜBITAK National Research Institute of Electronics and Cryptology

(Turkey); K. Yeğin, TÜBITAK National Research Institute of Electronics and Cryptology

(Turkey) and Yeditepe Univ. (Turkey); M. Sezgin, E. Bıçak, H. Belikli, H. Kara, L. Tura,

V. Özbudak, R. Gürcan, E. Özkan, M. Çayır, M. Dağ, TÜBITAK National Research Institute of Electronics and Cryptology (Turkey)

8714 OK Buried target radar imaging with an ultra-wideband, vehicle-mounted antenna array [8714-20]

K. Ranney, D. Liao, T. Dogaru, C. Tran, L. Nguyen, U.S. Army Research Lab. (United States)

$8714 \mathrm{OL}$ Three-dimensional radar imaging of buildings based on computer models [8714-21]

T. Dogaru, D. Liao, C. Le, U.S. Army Research Lab. (United States)

$87140 \mathrm{M}$ Spectral characteristics of human and indoor clutter for through the wall sensing [8714-22]

T. D. Bufler, R. M. Narayanan, The Pennsylvania State Univ. (United States); T. Dogaru,

U.S. Army Research Lab. (United States); E. H. Lenzing, The Pennsylvania State Univ. (United States) 
$8714 \mathrm{ON}$ Textural feature based target detection in through-the-wall radar imagery [8714-23]

A. Sengur, Firat Univ. (Turkey); M. Amin, F. Ahmad, Villanova Univ. (United States);

P. Sévigny, D. DiFilippo, Defence Research and Development Canada, Ottawa (Canada)

\section{SESSION 6 SPECIAL SESSION: MIMO RADAR}

871400 SIRE: a MIMO radar for landmine/IED detection [8714-24]

O. Ojowu Jr., Y. WU, J. Li, Univ. of Florida (United States); L. Nguyen, U.S. Army Research Lab. (United States)

8714 OP Robust adaptive beamforming for MIMO monopulse radar [8714-26]

W. Rowe, Univ. of Florida (United States); M. Ström, Chalmers Univ. of Technology

(Sweden); J. Li, Univ. of Florida (United States) and IAA, Inc. (United States); P. Stoica,

Uppsala Univ. (Sweden)

$87140 Q \quad$ New distributed radar technology based on UAV or UGV application [8714-27]

P. A. Molchanov, AMPAC, Inc. (United States); V. M. Contarino, R Cubed Inc. (United States)

$8714 \mathrm{OR}$ Tomographic imaging with ultra-wideband noise radar using time-domain data [8714-28] H. J. Shin, R. M. Narayanan, The Pennsylvania State Univ. (United States); M. Rangaswamy, Air Force Research Lab. (United States)

\section{SESSION 7 PROGRAMS AND SYSTEMS I}

8714 OS Concealed target detection using augmented reality with SIRE radar [8714-29]

P. Saponaro, C. Kambhamettu, Univ. of Delaware (United States); K. Ranney, A. Sullivan,

U.S. Army Research Lab. (United States)

8714 OT Lightweight SAR GMTI radar technology development [8714-30]

J. C. Kirk Jr., K. Lin, A. Gray, C. Hseih, S. Darden, W. Kwong, Goleta Star, LLC (United States);

U. Majumder, S. Scarborough, Air Force Research Lab. (United States)

8714 OU SAR and LIDAR fusion: experiments and applications [8714-31]

M. C. Edwards, E. C. Zaugg, J. P. Bradley, R. D. Bowden, ARTEMIS, Inc. (United States)

$87140 \mathrm{~V}$ High-efficiency switching power amplifiers for $P, L, S$, and $X$ Band [8714-32]

J. Lawler, J. Wells, S. Mendez, T. Wurth, NuWaves Engineering (United States)

\section{SESSION $8 \quad$ PROGRAMS AND SYSTEMS II}

8714 OW Calibration methods for phased array radars [8714-33]

I. Şeker, ASELSAN Inc. (Turkey)

8714 OX Cognitive nonlinear radar test-bed [8714-34]

A. S. Hedden, D. A. Wikner, A. Martone, D. McNamara, U.S. Army Research Lab. (United States) 
8714 OY Compact, autonomous, multi-mission synthetic aperture radar [8714-35]

T. J. Walls, M. L. Wilson, Naval Research Lab. (United States); D. Madsen, C. Knight,

M. D. Jensen, D. C. Partridge, Space Dynamics Lab. (United States); M. Addario, SRC Inc.

(United States)

$87140 Z$ Real-time beyond the horizon vessel detection [8714-36]

H. J. Roarty, M. Smith, S. M. Glenn, Rutgers Coastal Ocean Observation Lab. (United

States); D. E. Barrick, CODAR Ocean Sensors (United States)

871410 Remote concealed threat detection by novel classification algorithms applied to multi-polarimetric UWB radar [8714-37]

D. O'Reilly, N. Bowring, N. D. Rezgui, D. Andrews, S. Harmer, Manchester Metropolitan Univ. (United Kingdom)

\section{SESSION 9 ALGORITHMS AND PROCESSING I}

871411 Transforming optical image data into a SAR system's range-based image space [8714-38] H. Anglberger, R. Speck, H. Suess, German Aerospace Ctr. (Germany)

871412 Rejection of interference and near-field coupled LNA-noise on FPA-fed multibeam dish antennas using 3D analog filters [8714-39]

A. Madanayake, The Univ. of Akron (United States) and Univ. of Calgary (Canada); C. Wijenayake, The Univ. of Akron (United States); T. Gunarathne, L. Belostotski, Y. Xu, L. Bruton, Univ. of Calgary (Canada)

871413 A method to evaluate residual phase error for polar formatted synthetic aperture radar systems [8714-40]

C. Musgrove, R. Naething, Sandia National Labs. (United States)

871414 Analysis of SAR autofocus performance [8714-41]

R. M. Naething, R. D. West, Sandia National Labs. (United States)

871415 Target position and velocity estimation for track declaration using air-to-air radar [8714-42] G. Liu, J. Ramrus, General Atomics Aeronautical Systems, Inc. (United States)

871416 A global review of optronic synthetic aperture radar/ladar processing [8714-43] L. Marchese, M. Doucet, P. Bourqui, INO (Canada); B. Harnisch, M. Suess, ESA-ESTEC (Netherlands); M. Legros, N. Desnoyers, S. Turbide, S. Turgeon, L. Mercier, M. Savard, A. Martel, F. Châteauneuf, A. Bergeron, INO (Canada)

\section{SESSION $10 \quad$ ALGORITHMS AND PROCESSING II}

871417 Comparison of filtering and smoothing algorithms for airborne radar data [8714-44] B. Balaji, Defence Research and Development Canada, Ottawa (Canada); K. Wang, McDonald, Dettwiler and Associates Ltd. (Canada); A. Damini, Defence Research and Development Canada, Ottawa (Canada); M. Goulding, K. Hagen, McDonald, Dettwiler and Associates Ltd. (Canada) 
871418 Instantaneous frequency and time-frequency signature estimation using compressive sensing [8714-45]

B. Jokanović, Univ. of Montenegro (Montenegro) and Villanova Univ. (United States); M. Amin, Villanova Univ. (United States); S. Stanković, Univ. of Montenegro (Montenegro) and Villanova Univ. (United States)

871419 Riemannian mean and space-time adaptive processing using projection and inversion algorithms [8714-46]

B. Balaji, Defence Research and Development Canada, Ottawa (Canada); F. Barbaresco, Thales Air Systems S.A. (France)

8714 1B High-resolution computation of electrical field propagation in land mine detection [8714-18]

Y. A. Gryazin, Idaho State Univ. (United States)

SESSION 11 MICRO-DOPPLER RADAR I: JOINT SESSION BETWEEN CONFERENCES 8714 AND 8734

8714 1C Micro-doppler and vibrometry at millimeter and sub-millimeter wavelengths [8714-15]

D. A. Robertson, S. L. Cassidy, The Univ. of St Andrews (United Kingdom)

8714 1D Initial measurements of the angular velocity of walking humans using an active millimeter-wave correlation interferometer [8714-17]

K. S. Zilevu, Johns Hopkins Univ. Applied Physics Lab. (United States); K. L. Kammerman, Syracuse Univ. (United States); J. A. Nanzer, Johns Hopkins Univ. Applied Physics Lab. (United States)

8714 IE Micro-range micro-doppler for dismount classification [8714-16]

D. Tahmoush, U.S. Army Research Lab. (United States)

POSTER SESSION

8714 IF A comparison of interferometric SAR antenna options [8714-48]

A. W. Doerry, D. L. Bickel, Sandia National Labs. (United States)

$87141 G$ Random-phase radar waveforms with shaped spectrum [8714-49]

A. W. Doerry, Sandia National Labs. (United States); B. Marquette, General Atomics Aeronautical Systems, Inc. (United States)

$87141 \mathrm{H}$ Compound radar waveforms with multiple frames [8714-50]

A. W. Doerry, Sandia National Labs. (United States); B. Marquette, General Atomics Aeronautical Systems, Inc. (United States)

$87141 \mathrm{~J}$ A mathematical observation on synthetic aperture radar [8714-53]

Y. Cao, S. Li, J. Lopez, A. Martinez, Z. Qiao, The Univ. of Texas-Pan American (United States)

$87141 \mathrm{~K}$ Platform for research and education on ground penetrating radar [8714-54]

M. J. Salvador, V. Jimenez, R. G. Lopez, R. von Borries, The Univ. of Texas at El Paso (United States) 
$87141 \mathrm{~L}$ Lynx multi-mode SAR in support of NATO Unified Vision 2012 trial [8714-55]

R. Dunkel, T. Verge, R. Linnehan, General Atomics Aeronautical Systems, Inc. (United States); A. Doerry, Sandia National Labs. (United States)

$87141 \mathrm{M}$ Anisotropic model-based SAR processing [8714-56]

C. Knight, Space Dynamics Lab. (United States); J. Gunther, T. Moon, Utah State Univ. (United States)

Author Index 


\section{Conference Committee}

Symposium Chair

Kenneth R. Israel, Major General (USAF Retired) (United States)

Symposium Cochair

David A. Whelan, Boeing Defense, Space, and Security

(United States)

Conference Chairs

Kenneth I. Ranney, U.S. Army Research Laboratory (United States)

Armin Doerry, Sandia National Laboratories (United States)

Conference Program Committee

Fauzia Ahmad, Villanova University (United States)

Joseph C. Deroba, U.S. Army Communications-Electronics Research, Development and Engineering Center (United States)

Benjamin C. Flores, The University of Texas at El Paso (United States)

Mark Govoni, U.S. Army Research, Development and Engineering

Command (United States)

Majeed Hayat, The University of New Mexico (United States)

Chandra Kambhamettu, University of Delaware (United States)

Seong-Hwoon Kim, Raytheon Space \& Airborne Systems (United States)

James L. Kurtz, University of Florida (United States)

Changzhi Li, Texas Tech University (United States)

Jenshan Lin, University of Florida (United States)

David G. Long, Brigham Young University (United States)

Jia-Jih Lu, General Atomics Aeronautical Systems, Inc. (United States)

Neeraj Magotra, The University of New Mexico (United States)

Anthony F. Martone, U.S. Army Research Laboratory (United States)

George J. Moussally, Mirage Systems (United States)

Ram M. Narayanan, The Pennsylvania State University (United States)

Lam H. Nguyen, U.S. Army Research Laboratory (United States)

Hector A. Ochoa, The University of Texas at Tyler (United States)

Ann M. Raynal, Sandia National Laboratories (United States)

Jerry Silvious, U.S. Army Research Laboratory (United States)

Brian Smith, U.S. Army Armament Research, Development and Engineering Center (United States)

Helmut H. Suess, Deutsches Zentrum für Luft- und Raumfahrt e.V. (Germany) 
David Tahmoush, U.S. Army Research Laboratory (United States)

\section{Session Chairs}

1 Special Session: Noise Radar I

Ram M. Narayanan, The Pennsylvania State University (United States)

2 Special Session: Noise Radar II

Yan Zhang, University of Oklahoma (United States)

3 Applications: Concealed Targets I

Mark Govoni, U.S. Army Research, Development and Engineering Command (United States)

$4 \quad$ Emerging Technologies

Ann M. Raynal, Sandia National Laboratories (United States)

5 Applications: Concealed Targets II

Ann M. Raynal, Sandia National Laboratories (United States)

6 Special Session: MIMO Radar

Ram M. Narayanan, The Pennsylvania State University (United States)

7 Programs and Systems I

Seong-Hwoon Kim, Raytheon Space \& Airborne Systems (United States)

8 Programs and Systems II

Seong-Hwoon Kim, Raytheon Space \& Airborne Systems (United States)

9 Algorithms and Processing I

Ann M. Raynal, Sandia National Laboratories (United States)

10 Algorithms and Processing II Chandra Kambhamettu, University of Delaware (United States)

11 Micro-doppler Radar I: Joint Session between Conferences 8714 and 8734

Ram M. Narayanan, The Pennsylvania State University (United States)

12 Micro-doppler Radar II: Joint Session between Conferences 8714 and 8734

Ram M. Narayanan, The Pennsylvania State University (United States) 